\title{
Demanda Ambulatorial em Três Serviços da Rede Pública do Município do Rio de Janeiro, Brasil
}

\author{
A Survey of Demand for Medical Care in Three Public Health Clinics \\ in Rio de Janeiro, Brazil
}

\author{
Marilia S. Carvalho', Eleonora d'Orsï, Enirtes C. Prates ${ }^{3}$, \\ Wálria D. M. Toschỉ, Tizuko Shiraiwa', Tatiana P. Campos ${ }^{3}$ Érica Elß \\ Norma L. Garcia'a, Ana Paula Junqueira ${ }^{3}$, Simone A. Serrão ${ }^{3}$, Elda L. Tavares ${ }^{3}$
}

CARVALHO, M. S.; d'ORSI, E.; PRATES, E. C.; TOSCHI, W. D. M.; SHIRAIWA, T.; CAMPOS, T. P.; ELL, E.; GARCIA, N. L.; JUNQUEIRA, A. P.; SERRÃO, S. A. \& TAVARES, E. L. A. Survey of Demand for Medical Care in Three Public Health Clinics in Rio de Janeiro, Brazil. Cad. Saúde Públ., Rio de Janeiro, 10 (1): 17-29, Jan/Mar, 1994.

A sample survey was conducted in three public ambulatory medical care units in Rio de Janeiro in order to show the demographic and nosological aspects of the clientele as well as to evaluate the quality of clinic records. The sample included 2,029 patients registered in the health clinics and 3,980 consultations from October 1, 1990 to September 30, 1991. The diagnoses were coded according to the 9th revision of the International Classification of Diseases. Females accounted for about $60 \%$ of the sample, and more than a half were between 15 and 49 years of age.

Children under 10 years old made up $28 \%$ of the total. About $38 \%$ of the patients came from the poor areas of the city: the "favelas". The case files were poorly filled out, frequently lacking useful information. The most frequent cause was classified in the XVIo chapter of the ICD - illdefined causes, followed by respiratory, infectious, genitourinary and circulatory diseases. External causes predominated in one of the health clinics, which is an emergency service. There was a great demand for preventive measures, such as prenatal care and child care, even at the clinic that was traditionally devoted only to curative medicine. The most frequent specific diagnoses were upper respiratory tract infection, hypertension, gynecological problems, prenatal care, and skin diseases. There were no records for referrals to other health services. The poor quality of the medical records causes problems in terms of quality of health care. Use of such record-keeping systems, even while criticizing them, is essential to improve them.

Key words: Clinic Demand; Morbidity; Quality of Records

\section{INTRODUÇÃO}

Há um interesse crescente no conhecimento do perfil epidemiológico para a adequação das

\footnotetext{
${ }^{1}$ Departamento de epidemiologia e Métodos Quantitativos em Saúde Pública da Escola Nacional de Saúde Pública. Rua Leopoldo Bulhões 1480, Rio de Janeiro, RJ, 21041-210, Brasil.

${ }^{2}$ Programa de Apoio a Reforma Sanitária. Escola Nacional de Saúde Pública. Rua Leopoldo Bulhões 1480 , $3^{\circ}$ andar, Rio de Janeiro, RJ, 21041-210, Brasil.

${ }^{3}$ Residência em Saúde Pública. Escola Nacional de Saúde Pública. Rua Leopoldo Bulhões 1480, $3^{\circ}$ andar, sala 317, Rio de Janeiro, RJ, 21041-210, Brasil.
}

práticas de saúde. Portanto, conhecer a demanda ambulatorial na rede pública tornou-se tarefa necessária tanto para a avaliação de serviços como para a orientação dos que trabalham com gerência, programação e planejamento em saúde (Radaelli et al., 1990).

Além disso, as análises de mortalidade, já bastante difundidas, que tradicionalmente subsidiam o planejamento em saúde devem ser complementadas pela análise da morbidade, pois há um grande número de doenças e agravos à saúde que não causam o óbito, mas levam a graus variados de espoliação.

Para se conhecer a morbidade na população 
são necessários tantos dados primários, oriundos de inquéritos domiciliares, nos locais de trabalho, escolas, etc., quanto dados secundários, disponíveis em prontuários de hospitais, ambulatórios, emergências, clínicas e consultórios, sejam eles públicos ou privados. Cada uma dessas abordagens permite que sejam visualizados alguns aspectos da realidade (Kostrzewski, 1982). Neste trabalho estudou-se a demanda ambulatorial de três unidades da rede pública da Área Programática (AP) 3.1 do município do Rio de Janeiro.

Os estudos de demanda servem basicamente a quatro objetivos: avaliar a qualidade dos registros em prontuário; verificar a adesão do serviço às normas preconizadas; avaliar o próprio serviço, na medida em que a qualidade dos registros permita; e, finalmente, inferior a respeito da população de origem da clientela assistida.

O presente trabalho faz parte de um projeto mais amplo, apoiado pelo Programa de Apoio à Reforma Sanitária, de inserção do Curso de Residência em Saúde Pública da Escola Nacional de Saúde Pública (Ensp), Fundação Oswaldo Cruz (Fiocruz), junto à rede de serviços de saúde da AP3.1. Os três serviços investigados concentram-se na X Região Administrativa (RA) Ramos -, que é parte da AP3.1, pois análises anteriores realizadas (Carvalho et al., 1993a) localizaram condições de saúde mais precárias nesta região.

A população de Ramos é de 288.313 habitantes (Fibge, 1991). A rede pública de serviços de saúde é composta por um hospital geral de grande porte, com emergência, mantido pelo Instituto Nacional de Assistência Médica da Previdência Social (Inamps); um hospital especializado em infectologia; um ambulatório especializado em hanseníase; um centro de saúde escola do Ministério da Saúde/Fiocruz; e uma unidade de cuidados básicos, mantida pela Universidade Federal do Rio de Janeiro. Foram selecionados para este estudo um posto de assistência médica, com setor ambulatorial e setor de urgência, denominados PAM-Ramos e PU-Ramos, mantido pelo Inamps, e um centro municipal de saúde (CMS-Ramos), mantido pela Secretaria Municipal de Saúde do Rio de Janeiro (SMS-RJ).

As unidades foram escolhidas por pertencerem ao setor público, prioridade da direção do sistema local, e por permitirem que fossem visuali- zados três modelos diferentes de atendimento. O CMS-Ramos é o centro de referência em atenção primária, porém está localizado em um dos extremos da região - no interior da favela da praia de Ramos -, ficando separado da maior concentração populacional por uma via de alta velocidade e pela via férrea. Oferece atendimento em diversas especialidades, além daquelas básicas, como Tisio-Pneumologia, Dermatologia e Psicologia, o que condicionará a demanda.

O PAM-Ramos localiza-se do outro lado da via expressa. Além das especialidades básicas, conta com Cardiologia, Psiquiatria, Alergia, Medicina Física e Fisioterapia. O setor de urgência (PU-Ramos), situado em outro prédio, é uma unidade de pronto atendimento, dispondo de ambulância para remoção dos casos mais graves. Considerando-se sua localização distinta e o modelo diferenciado de atendimento, o PAMRamos e o PU-Ramos foram analisados em separado. O PU-Ramos é o serviço mais próximo da maior favela da região.

Ao se realizar este trabalho, a intenção foi, além de instrumentalizar os serviços de saúde da região, buscar formas para facilitar a utilização desta metodologia nos serviços de maneira geral. Este objetivo foi alcançado, em parte, pela publicação de um texto onde é descrito cada passo desta empreitada (Carvalho et al., 1993b). Uma vez que este artigo também se insere nesta perspectiva, optou-se por apresentar toda a variedade de resultados obtidos, ao invés de priorizar um tema e aprofundá-lo. Sem dúvida, os dados coletados sobre alguns aspectos permitem e merecem uma análise mais aprofundada, o que ainda será feito.

\section{OBJETIVOS}

Este trabalho teve por objetivo avaliar a qualidade dos registros do prontuário, verificar a adesão do serviço a normas preconizadas de préconsulta, e traçar o perfil demográfico e nosológico da clientela dos serviços estudados.

\section{METODOLOGIA}

Foram incluídos no universo os prontuários com registro de consultas médicas, de enfermagem e de nutrição entre 01 de outubro de 1990 e 30 de setembro de 1991. Analisou-se o perfil da clientela através dos dados de identificação e 
de todas as consultas do período registradas nos prontuários da amostra.

A amostra estimada em cada serviço foi de 540 prontuários ou fichas, admitindo-se um erro de $5 \%$. Entretanto, devido às particularidades de cada arquivo, a amostra de fato obtida variou, sendo de 353 prontuários, com um total de 701 consultas, no CMS-Ramos e de 1.082 prontuários, com 2.685 consultas, no PAM-Ramos. No PU-Ramos, onde o sistema de registro é através de fichas de pronto atendimento, cada ficha correspondendo a um atendimento, a amostra obtida foi de 594 fichas. Em todos os prontuários amostrados, cerca de $97 \%$ das consultas eram médicas, inviabilizando, assim, a análise das consultas feitas por enfermeiros ou nutricionistas, conforme a proposta original.

Os diagnósticos foram codificados pela $9^{\mathrm{a}}$ revisão da Classificação Internacional de Doenças - CID (OMS, 1978). Foram codificados até três diagnósticos por consulta. Não foi possível trabalhar com o motivo de consulta por falta de registros. Na codificação dos diagnósticos, optou-se por aproveitar todo registro que pudesse trazer informação diagnóstica, mesmo que este não estivesse escrito, resgatando-se a informação contida em registros de sinais, sintomas e/ou conduta, quando estes deixavam evidente um diagnóstico implícito, e em registros de consultas anteriores, quando o assinalado era "conduta mantida". Nestes casos, o diagnóstico codificado recebia o código "presumido pelo codificador".

Os endereços foram classificados por favela e bairro (Guia Quatro Rodas, 1990; Iplanrio, 1982).

O trabalho de campo foi realizado por seis alunos do $1^{\circ}$ ano do Curso de Residência Multiprofissional da Ensp entre 8 de outubro e 20 de dezembro de 1991. A codificação e supervisão ficaram centralizadas com duas monitoras do $2^{\circ}$ ano da Residência. $\mathrm{O}$ trabalho foi coordenado por uma professora do Departamento de Epidemiologia e Métodos Quantitativos em Saúde da Ensp.

Foram utilizados microcomputadores IBM/ $\mathrm{XT}$, gerenciador de banco de dados Dbase III Plus (Ashton-Tate, 1985), pacote estatístico Epi-Info (Dean, 1990) e, no desenho dos gráficos, o Harvard Graphics (SPC, 1990).

\section{RESULTADOS E DISCUSSÃO}

\section{Perfil Demográfico}

Nas três unidades predominou o atendimento aos menores de 10 anos e mulheres na faixa etária reprodutiva. A proporção de homens no PU-Ramos aproxima-se de 50\% (Figura 1). A concentração de consultas, ou seja, média de consultas por pessoa por ano, é maior no CMSRamos $(4,28)$ do que no PAM-Ramos $(2,66)$. Analisando-se este indicador de aderência ao serviço em cada faixa etária, observou-se maior freqüência dos menores de 1 ano de idosos em ambos os serviços. No grupo de 15 a 64 anos, a concentração de consultas é maior nas mulheres (Tabela 1).

Estes resultados são comuns a outros estudos (Benitez \& Monzón, 1985; Deloizer \& Gagnon, 1991; Tanaka \& Rosemburg, 1990; YazzeleRocha \& Nogueira, 1985), provavelmente condicionados pela prioridade dada à atenção à infância e pela menor inserção da mulher no mercado formal de trabalho, com maior disponibilidade de tempo, além de uma possível percepção diferenciada do seu processo saúdedoença. Por outro lado, o horário de funcionamento e a oferta de atendimento não facilitam o acesso da população masculina adulta ao serviço. Estes motivos, acrescidos da maior freqüência de problemas de saúde, geram um novo aumento na concentração de consultas na terceira idade. No PU-Ramos, onde o atendimento é feito em regime de plantão, sem marcação prévia, a procura de homens é maior, aproximadamente 50\% da demanda. Entretanto, a forma de registro, através de ficha de pronto atendimento, inviabiliza a análise do retorno do paciente ao serviço e, portanto, o cálculo da concentração de consultas.

Ao se analisar a distribuição da demanda segundo moradia em favela ou área urbanizada, observa-se que, no PU-Ramos, $45 \%$ provêm de área urbanizada e $20 \%$ de favelas; no PAMRamos, 30\% vêm de área urbanizada e 39\% de favelas; e no CMS-Ramos, apenas $12 \%$ vêm de área urbanizada, sendo bem maior a freqüência de população favelada (60\%). Isto pode ser explicado pela localização das unidades e pelo grande percentual (cerca de 50\%) de moradores em favelas nesta RA (Fibge, 1980). Não foi 
FIGURA 1. Perfil Demográfico da Clientela por Unidade, Estudo de Demanda, Outubro de 1990 a Setembro de 1991

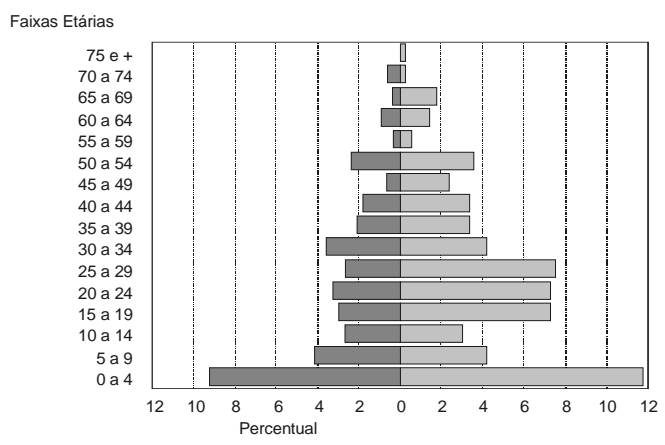

PAM-RAMOS

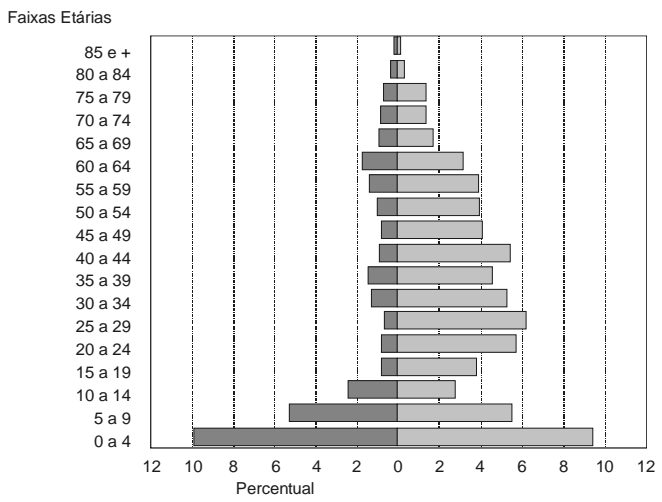

PU-RAMOS

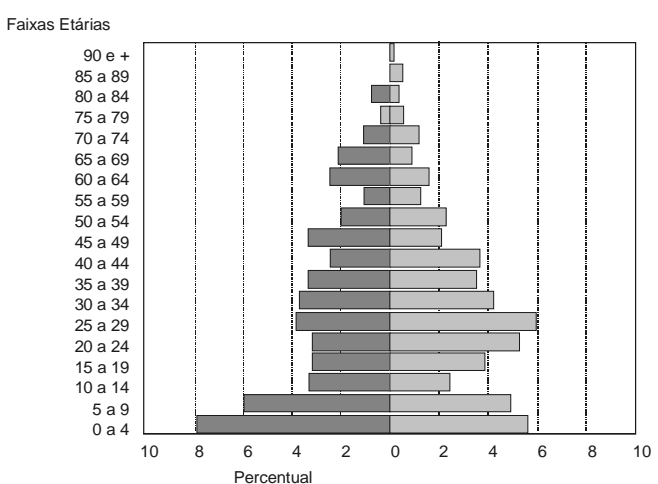

possível localizar cerca de $30 \%$ dos endereços, que acredita-se serem ruas não-cadastradas de favelas (Figura 2). O mapeamento indica uma forte concentração da demanda em torno da unidade no CMS-Ramos e um espalhamento maior no PU-Ramos, mas, mesmo assim, em todas as unidades, mais de $80 \%$ da população assistida mora na X RA (Figura 3).

TABELA 1. Concentração de Consultas por Faixa Etária, Sexo e Unidade, Estudo de Demanda, Outubro de 1990 a Setembro de 1991

\begin{tabular}{|c|c|c|c|}
\hline Faixas Etárias & CMS & PAM & Total \\
\hline$<1$ & 5,25 & 3,2 & 4,22 \\
\hline 1 a 14 & 2,8 & 2,16 & 2,48 \\
\hline masc. & 3,01 & 2,43 & 2,72 \\
\hline \multicolumn{4}{|l|}{15 a 64} \\
\hline fem. & 4,49 & 2,6 & 3,54 \\
\hline $65 \mathrm{e}+$ & 5,85 & 2,94 & 4,39 \\
\hline Total & 4,28 & 2,66 & 3,47 \\
\hline
\end{tabular}

FIGURA 2. Classificação da Demanda segundo Moradia em Favela ou Área Urbanizada por Unidade, Estudo de Demanda, Outubro de 1990 a Setembro de 1991.

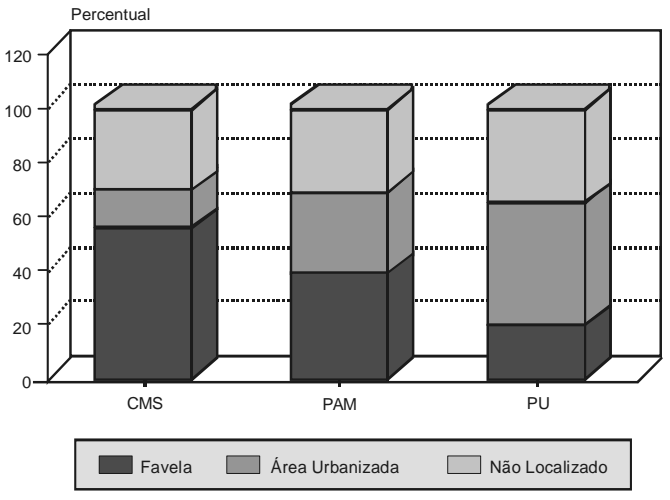


FIGURA 3. Origem da Clientela por Unidade, Estudo de Demanda, Outubro de 1990 a Setembro de 1991

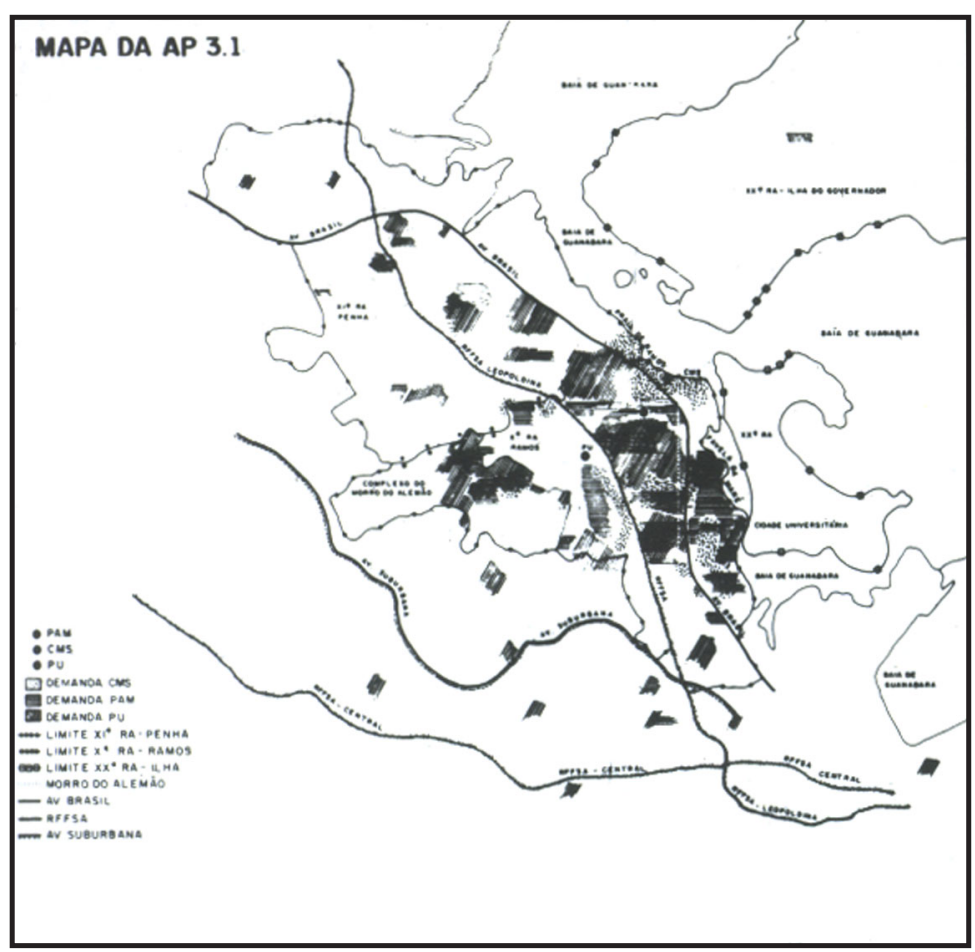

\section{Registros}

Nos prontuários do CMS-Ramos, $38 \%$ das consultas não apresentavam carimbo, assinatura ou rubrica de identificação do profissional que realizou a consulta. $\mathrm{O}$ mesmo aconteceu no PAM-Ramos em $22 \%$ das consultas. Este indicador, relacionado à responsabilidade do profissional sobre sua atividade, indicou uma substancial indiferença do profissional, neste caso o médico, em relação ao registro da consulta e à clientela atendida. No PU-Ramos, as fichas não assinadas pelo médico que atendeu ao caso são assinadas pelo chefe do plantão ao final do dia, retirando-se deste indicador sua função na avaliação da qualidade de registro.

Nos três serviços, o registro mais freqüentemente encontrado na consulta médica foi o da conduta adotada pelo médico, presente em cerca de $85 \%$ das consultas. O cuidado com o registro da queixa e/ou história da doença foi menor; em média, apenas $67 \%$ das consultas o apresentavam. Em apenas $38 \%$ das consultas houve registro de exame físico. Quanto ao diagnóstico, o PU-Ramos se destaca das demais unidades, com $73 \%$ de registros presentes (Figura 4). Isto se deve, provavelmente, à presença de espaço próprio para este registro na ficha de atendimento desta unidade, com um quadro especificamente para este registro. Também o caráter agudo das patologias ali atendidas favorece o estabelecimento de um diagnóstico mais preciso em apenas uma consulta. É importante ressaltar que, em unidades ambulatoriais, é esperado um percentual de atendimentos sem diagnóstico ou com patologias maldefinidas.

É importante observar que a presença destes registros na consulta médica não tem implicações diretas na qualidade da consulta médica, uma vez que os procedimentos podem ter sido realizados, embora não registrados. Entretanto, a ausência de registro tem implicações sérias no seguimento do paciente e reflete uma concepção muito comum no meio médico, isto é, de que registro é "burocracia", sendo secundário frente às atividades junto ao paciente. 
Ao se analisar a presença de registro de pressão arterial nas consultas de pacientes acima de 30 anos de idade, nota-se notamos que esse percentual variou em 50\% no CMSRamos, 35\% no PAM-Ramos e 30\% no PURamos. Levando-se em conta a alta prevalência de hipertensão arterial (cerca de 20\%) obser- vada nesta faixa etária e as normas do Ministério da Saúde, que preconizam a aferição da pressão arterial em todos os indivíduos de 20 anos ou mais que procurem o serviço de saúde por qualquer razão (MS, 1988), os percentuais encontrados são considerados muito aquém do ideal.

FIGURA 4. Presença de Registros da Consulta Médica por Tipo de Registro e Unidade, Estudo de Demanda, Outubro de 1990 a Setembro de 1991
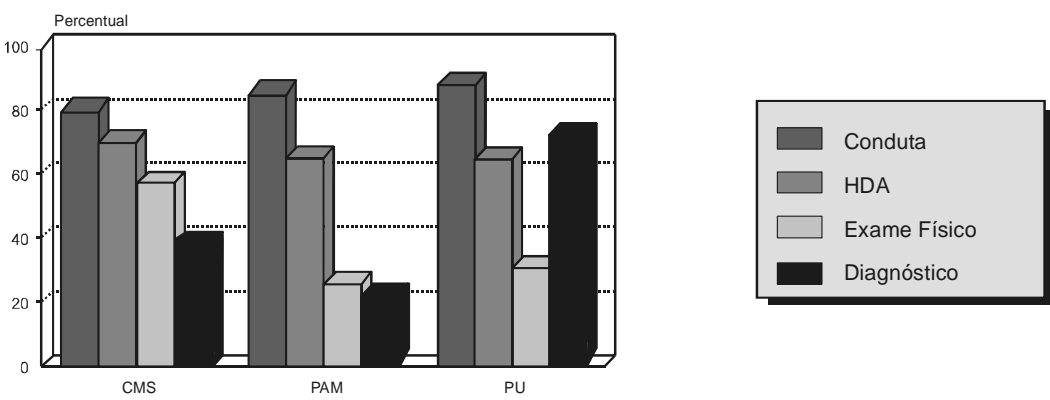

Já o registro de peso em consultas de menores de 5 anos ficou em torno de $80 \%$ nos serviços ambulatoriais. Na faixa etária de 5 a 14 anos houve registro em $22 \%$ no CMSRamos e em $75 \%$ no PAM-Ramos, caindo abruptamente a partir dessas idades e sendo nulo acima de 50 anos (Figura 5). No PURamos, a pesagem não faz parte da rotina. Estes dados apontam para uma defasagem em relação ao cumprimento das normas do Progra- ma de Atenção Integral à Saúde da Criança, que preconiza o acompanhamento do crescimento e desenvolvimento dos menores de 5 anos em todos os comparecimentos da criança à unidade (MS, 1984). Cabe também refletir sobre a questão do controle da obesidade em adultos, fator de risco comprovado para muitas doenças, entre elas as cardiovasculares, de elevada prevalência na população atendida nestes serviços.

FIGURA 5. Presença de Registros de Peso por Faixa etária e Unidade, Estudo de Demanda, Outubro de 1990 a Setembro de 1991

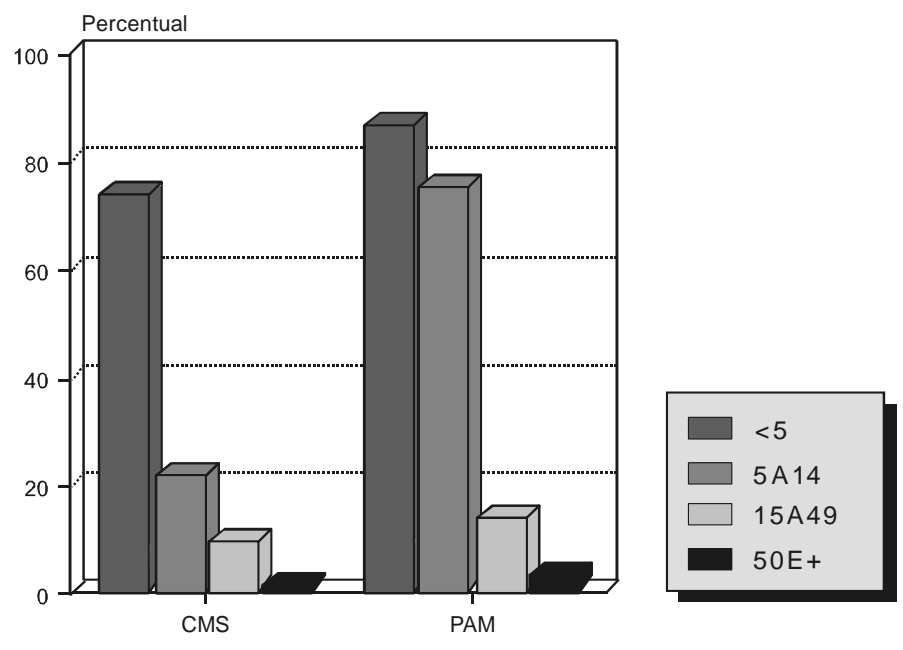


Os percentuais encontrados de registros de referência, exames solicitados/realizados, procedimentos e imunizações foram muito baixos. Existe uma rotina de referência de pacientes do PU-Ramos para o PAM-Ramos, uma vez que o PU-Ramos não se propõe a dar seguimento aos pacientes atendidos. Contudo, não se encontrou registro deste procedimento nas consultas.

\section{Perfil Nosológico}

O número médio de diagnósticos por consulta foi de 1,12 no CMS-Ramos, 0,91 no PAMRamos e 1,01 no PU-Ramos. Frente ao número relativamente reduzido de consultas com mais de um diagnóstico, optou-se por trabalhar com apenas o primeiro diagnóstico de cada consulta, selecionado por ser o mais preciso, Explicitamente escritos no prontuário foram encontrados 59\% dos diagnósticos no CMS-Ramos, $51 \%$ no PAM-Ramos e $79 \%$ no PU-Ramos. Dos restantes, foi possível presumir, a partir de sinais, sintomas ou medicamentos prescritos, cerca de $31 \%$ dos diagnósticos nos serviços ambulatoriais e $9 \%$ no serviço de urgência. Cerca de $11 \%$ dos diagnósticos estavam ilegíveis e $2 \%$ estavam sem registro nos três serviços (Figura 6).

FIGURA 6. Perfil de Registro do Primeiro Diagnóstico por Unidade, Estudo de Demanda, Outubro de 1990 a Setembro de 1991

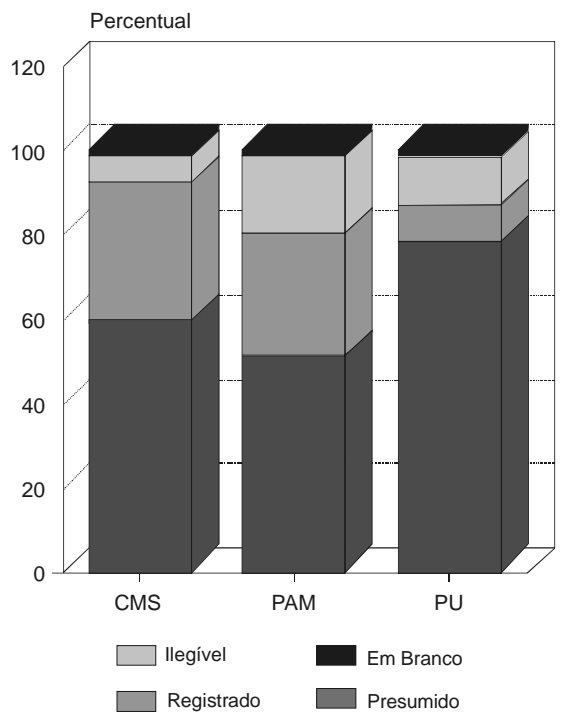

A classificação por grandes grupos da CID (Classificação Internacional de Doenças) mostrou que, nos serviços ambulatoriais, há um percentual significativo de diagnósticos relacionados à "Classificação suplementar de fatores que exercem influência sobre o estado de saúde e de oportunidades de contato com o serviço de saúde" (código "V"). Também são freqüentes consultas sem diagnósticos, principalmente no PAM-Ramos (20\%). Cerca de 14\% dos diagnósticos situam-se no capítulo dos "Sintomas, sinais e afecções maldefinidas" nos três serviços. Esta questão pode estar ligada à característica do serviço ambulatorial, que funciona como porta de entrada, onde o paciente ainda não foi submetido a uma investigação mais detalhada. Ela pode também ser reflexo da baixa resolutividade do atendimento.

Entre as causas que puderem ser classificadas, os capítulos da CID que aparecem com maior frequiência no CMS-Ramos são: "Doenças infecciosas e parasitárias", com 23\% dos diagnósticos; "Doenças do aparelho respiratório, com 15\%; e "Doenças do aparelho geniturinário, também com $15 \%$. O PAM-Ramos apresenta uma freqüência maior de diagnósticos nos capítulos das "Doenças do aparelho respiratório" (12\%) e "Doenças do aparelho circulatório" (12\%), destacando-se também o capítulo dos "Transtornos mentais" (9\%), este último provavelmente devido à oferta de atendimento em Psiquiatria. No PU-Ramos destaca-se o capítulo das "Doenças do aparelho respiratório" (24\%), seguido pelo das "Doenças infecciosas e parasitárias" (19\%). O capítulo de "causas externas e classificação suplementar 'E'” aparece com maior frequiência no PU-Ramos (11\%) do que nos outros dois serviços (Tabela 2).

A seguir são apresentados alguns agrupamentos de causas correlatas mais detalhados do que os capítulos da CID, selecionados em função da freqüência encontrada e de alguns programas do Ministério da Saúde, como as ações normatizadas de assistência à criança e ao hipertenso. As respectivas definições encontram-se no Quadro 1.

O perfil de atendimento em menores de 5 anos está bem definido pelas principais ações do programa de assistência à saúde da criança (MS, 1984): infecções respiratórias agudas e diarréias (Figura 7). Os problemas ginecológi- 
cos e as consultas de pré-natal, juntos, são a maior causa de procura ao serviço entre mulheres na faixa etária reprodutiva, tanto no CMS-Ramos, onde são responsáveis por $25 \%$ das consultas, como no PAM-Ramos, com 35\% do total (Figura 8). Estes dados apontam para a existência de um modelo assistencial principalmente voltado para a atenção materno-infantil. Os homens adultos pouco comparecem aos serviços. Quando o fazem, a procura é principalmente por problemas psicológicos e neuro- lógicos, no PAM-Ramos (44\%), e por causas externas, no PU-Ramos (15\%). Nota-se também notamos um percentual expressivo de problemas classificados no capítulo de "Sintomas e sinais maldefinidos" (cerca de 16\%) entre homens de 15 a 49 anos nos três serviços (Figura 9). A hipertensão arterial é a principal causa de demanda nos três serviços na faixa etária acima de 50 anos, sendo responsável por $42,7 \%$ das consultas no CMS-Ramos, $28,9 \%$ no PAMRamos e 24,4\% no PU-Ramos (Figura 10).

TABELA 2. Distribuição dos Primeiros Diagnósticos segundo Capítulos da CID (9a Revisão) por Unidade, Estudo de Demanda, Outubro de 1990 a Setembro de 1991

\begin{tabular}{|c|c|c|c|c|c|c|}
\hline \multirow{2}{*}{$\begin{array}{l}\text { Capítulos } \\
\text { da CID }\end{array}$} & \multicolumn{2}{|c|}{ CMS } & \multicolumn{2}{|c|}{ PAM } & \multicolumn{2}{|c|}{ PU } \\
\hline & $\mathrm{N}$ & $\%$ & $\mathrm{~N}$ & $\%$ & $\mathrm{~N}$ & $\%$ \\
\hline I. DIP & 150 & 23,1 & 196 & 9,1 & 100 & 19,4 \\
\hline II. Neoplasias & 7 & 1,1 & 6 & 0,3 & 1 & 0,2 \\
\hline III. Metabólicas & 5 & 0,8 & 40 & 1,9 & 2 & 0,4 \\
\hline IV. Sangue & 9 & 1,4 & 15 & 0,7 & 0 & 0,0 \\
\hline V. Mentais & 7 & 1,1 & 185 & 8,6 & 11 & 2,1 \\
\hline VI. Sistema Nervoso & 4 & 0,6 & 51 & 2,4 & 12 & 2,3 \\
\hline VII. Circulatório & 60 & 9,2 & 257 & 11,9 & 45 & 8,7 \\
\hline VIII. Respiratório & 99 & 15,2 & 269 & 12,5 & 126 & 24,5 \\
\hline IX. Digestivo & 9 & 1,4 & 39 & 1,8 & 15 & 2,9 \\
\hline X. Geniturinário & 98 & 15,1 & 228 & 10,6 & 24 & 4,7 \\
\hline XI. Gravidez & 5 & 0,8 & 1 & 0,0 & 10 & 1,9 \\
\hline XII. Pele & 27 & 4,2 & 28 & 1,3 & 5 & 1,0 \\
\hline XIII. Osteomuscular & 0 & 0,0 & 114 & 5,3 & 22 & 4,3 \\
\hline XIV. Congênitas & 0 & 0,0 & 1 & 0,0 & 0 & 0,0 \\
\hline XV. Perinatais & 21 & 3,2 & 6 & 0,3 & 0 & 0,0 \\
\hline XVI. Maldefinidas & 92 & 14,2 & 307 & 14,2 & 70 & 13,6 \\
\hline XVII. Externas* & 8 & 1,2 & 29 & 1,3 & 58 & 11,3 \\
\hline Serviços de Saúde*** & 49 & 7,5 & 383 & 17,8 & 14 & 2,7 \\
\hline Subtotal & 650 & 100,0 & 2155 & 100,0 & 515 & 100,0 \\
\hline Sem Diagnóstico*** & 51 & 7,3 & 530 & 19,7 & 70 & 12,0 \\
\hline Total & 701 & 100,0 & 2685 & 100,0 & 585 & 100,0 \\
\hline
\end{tabular}

\footnotetext{
* Incluindo Classificação Suplementar "E"

** Classificação Suplementar "V"

*** Consultas cujo registro de diagnóstico foi ilegível ou em branco
} 
FIGURA 7. Distribuição dos Agrupamentos de Diagnósticos em Menores de 5 anos por Unidade, Estudo de Demanda, Outubro de 1990 a Setembro de 1991

PAM-RAMOS

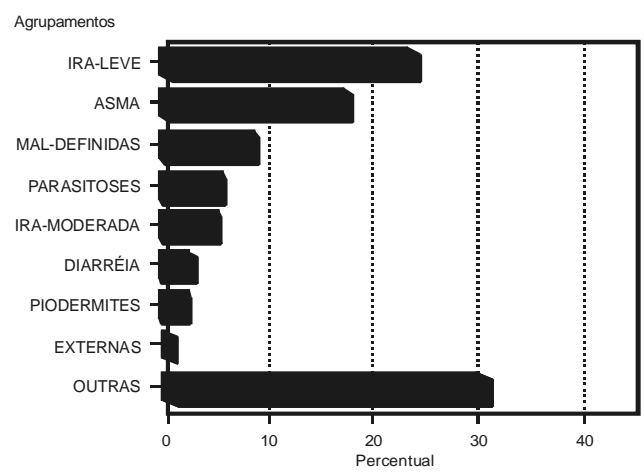

CMS-RAMOS

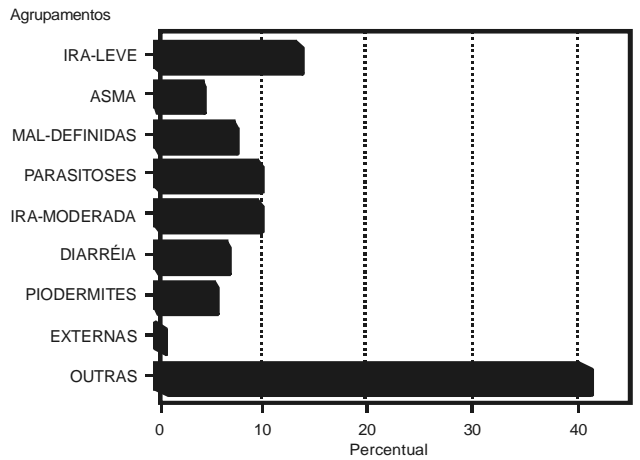

PU-RAMOS

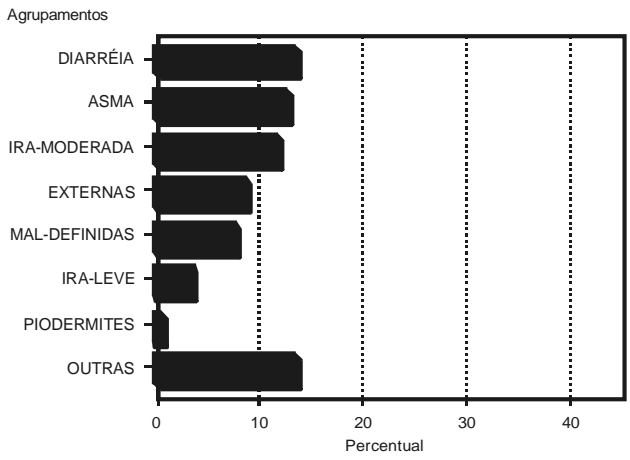

FIGURA 8. Distribuição dos Agrupamentos de Diagnósticos em Mulheres de 15 a 49 anos por Unidade, Estudo de Demanda, Outubro de 1990 a Setembro de 1991

PAM-RAMOS

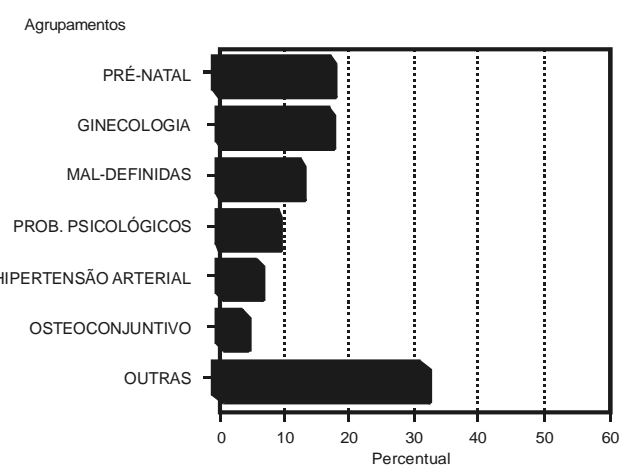

CMS-RAMOS
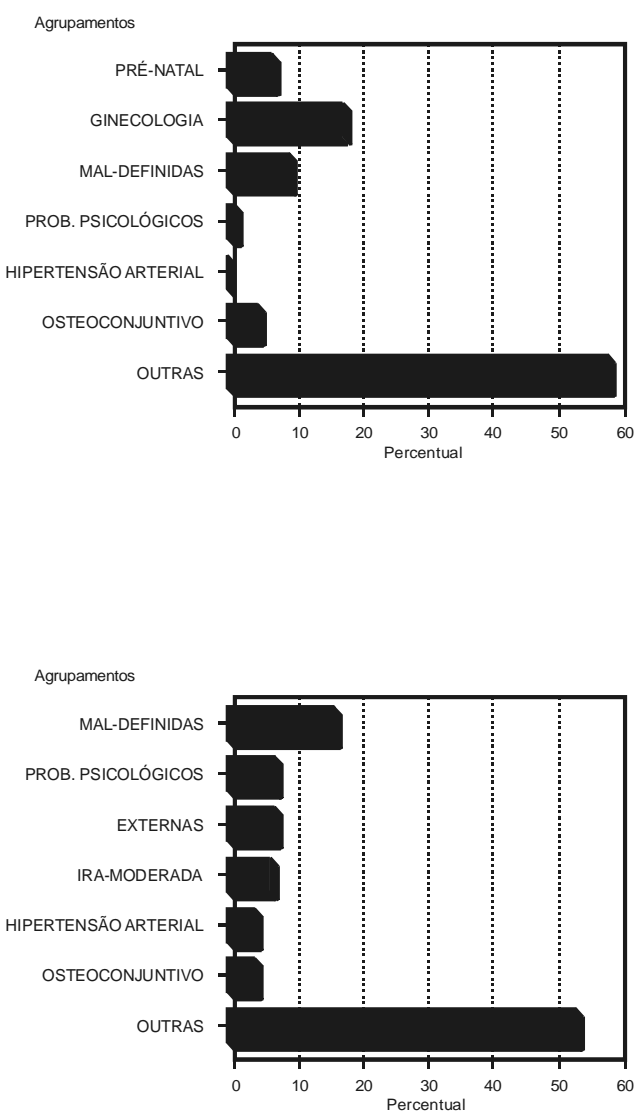
QUADRO 1. Agrupamentos de Diagnósticos Criados com as respectivas CIDs, Estudo de Demanda, Outubro de 1990 a Setembro de 1991

\begin{tabular}{|c|c|}
\hline CATEGORIA & CIDs \\
\hline Asma & - asmas, bronquites, rinites \\
\hline Diarréia & $\begin{array}{l}\text { - infecção intestinal por E. coli, colite, gastroenterite infecciosa presumível, diarréia } \\
\text { infecciosa presumível, salmoneloses }\end{array}$ \\
\hline Eczemas & - dermatites atópicas, dermatites de contato, e não especificadas \\
\hline Externas & - capítulo XVII da CID e classificação suplementar E \\
\hline Febre & - hipertermia de origem desconhecida \\
\hline Ginecologia & $\begin{array}{l}\text { - doenças da mama, dispaurenia, dismenorréia, amenorréia, metrorragia, colpite, } \\
\text { vaginite, abscesso de bartholin }\end{array}$ \\
\hline Hipertensão & • hipertensão arterial essencial \\
\hline Ira-Leve & - faringites, resfriado, tosse, ira não especificada \\
\hline Ira-Moderada & $\begin{array}{l}\text { - amigdalites purulentas, pneumonias sem insuficiência respiratória, otites, } \\
\text { bronquite aguda }\end{array}$ \\
\hline Maldefinidas & $\begin{array}{l}\text { - Sintomas maldefinidos, por exemplo: convulsões, tonteira, hipertermia, } \\
\text { mal-estar, edema, anorexia, cefaléia, dispnéia, dor torácica, azia, ascite, } \\
\text { nervosismo, distúrbios do sono, ganho ou perda anormal de peso, etc. }\end{array}$ \\
\hline Neurologia & - epilepsia, enxaqueca, paralisia do nervo facial \\
\hline Osteoconjuntivo & - colagenoses, artroses, artrites, lumbago, ciática, entesopatias, mialgias, cisto sinovial \\
\hline Parasitoses & $\begin{array}{l}\text { - parasitose intestinal não especificada, amebíase, teníase, ancilostomíase, } \\
\text { ascaridíase, enterobíase, giardíase }\end{array}$ \\
\hline Piodermites & - furúnculo, abscessos, pioderma \\
\hline Preventivo & - preventivo de câncer uterino \\
\hline Problemas & \\
\hline Psicológicos & - alcoolismo, delirium tremens, psicoses, ansiedade, histeria, depressão, neurose \\
\hline Virose & • viroses não especificadas \\
\hline
\end{tabular}

FIGURA 9. Distribuição dos Agrupamentos de Diagnósticos em Homens de 15 a 49 anos por Unidade, Estudo de Demanda, Outubro de 1990 a Setembro de 1991

PAM-RAMOS

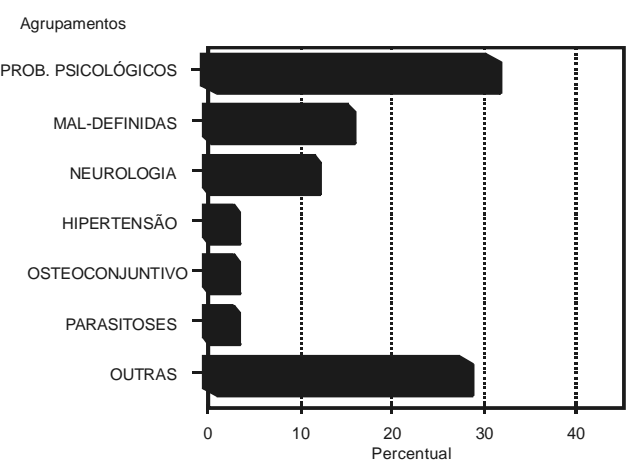

Ao se avaliar as causas mais freqüentes isoladas por serviço, observa-se que a hipertensão arterial e as doenças do aparelho respiratório têm um peso importante nos três serviços. No PAM-Ramos nota-se uma maior presença de consultas por ações preventivas, como prénatal, puericultura e exame preventivo de
PU-RAMOS

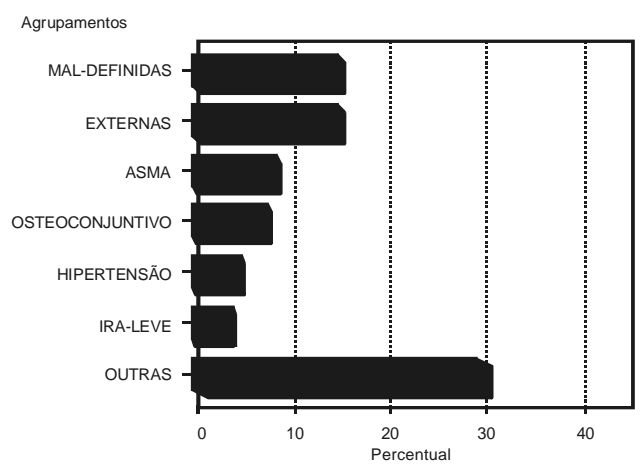

câncer ginecológico. No CMS-Ramos, as doenças de pele aparecem com freqüência. No PURamos, como era esperado, por ser um serviço de urgência, são mais freqüentes as causas externas e também outras afecções que requerem atendimento urgente, como virose aguda, febre, entre outras (Quadro 2). 
FIGURA 10. Distribuição dos Agrupamentos de Diagnósticos em Pacientes de 50 anos ou mais por Unidade, Estudo de Demanda, Outubro de 1990 a Setembro de 1991

PAM-RAMOS

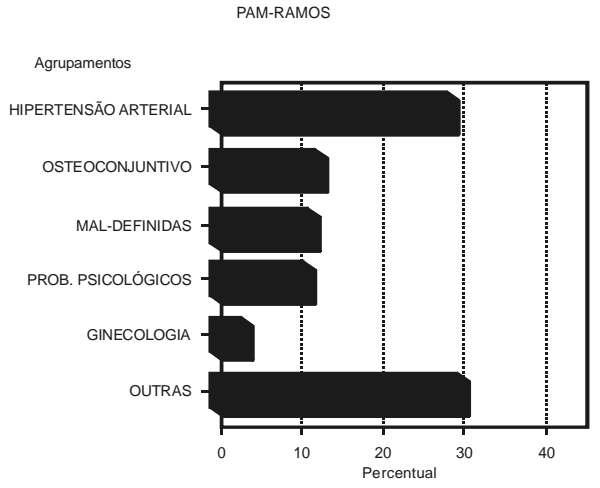

CMS-RAMOS

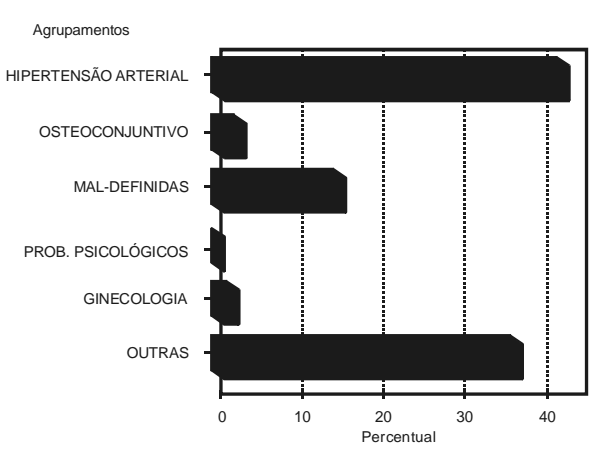

PU-RAMOS

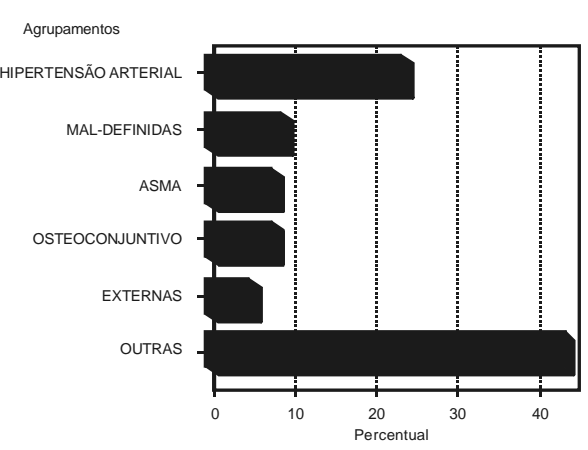

\section{CONCLUSÕES}

Os serviços estudados atendem basicamente à população favelada. Acredita-se que, até certo ponto, o fato de atenderem predominantemente à população favelada contribui para a visão da atenção primária em saúde como atenção de segunda classe para cidadãos de segunda classe. Esta mentalidade funciona como empecilho à incorporação de tecnologias pelos serviços, cujas necessidades vão das mais simples, como pesagem e aferição da pressão arterial, às de média complexidade, como exames complementares laboratoriais, pouco encontrados nos registros em prontuários. Dar prioridade a essas questões é uma condição fundamental para se melhorar a resolutividade do atendimento.

Neste trabalho observa-se uma mudança nos perfis assistenciais do CMS-Ramos, que tradicionalmente realizava ações preventivas, e do PAM-Ramos, que historicamente ficava com as ações curativas. Atualmente, estes têm perfis semelhantes: o PAM-Ramos passou a oferecer ações na área materno-infantil e o CMS-Ramos incorporou a assistência médica, embora ainda com predomínio da clientela de mulheres e crianças. Por outro lado, o PU-Ramos, a princípio voltado para o atendimento de urgência, atende a uma expressiva demanda ambulatorial.

Existe uma aderência natural da clientela, condicionada pela localização geográfica dos serviços, o que aponta para a viabilidade da regionalização.

A qualidade dos registros médicos em prontuário mostrou-se extremamente precária. Considera-se importante a melhoria destes registros, como forma de garantir a continuidade da assistência ao paciente, possibilitar a avaliação da qualidade do atendimento prestado, bem como sua resolutividade, além de ser fundamental para a gerência do serviço. Acredita-se que um elemento fundamental para a melhoria destes registros é sua utilização, ainda que apontando os problemas.

A demanda analisada é condicionada pela oferta de especialidades de cada serviço, conforme observado pela alta frequiência de causas mentais no PAM-Ramos; causas dermatológicas no CMS-Ramos; asma e causas externas no PU-Ramos. Evidentemente, a inexistência de 
determinadas especialidades nos serviços estudados implica uma demanda específica reprimida. Pouco se sabe acerca da adequação qualitativa e quantitativa desta oferta ao perfil nosológico da região, o que já seria outro estudo.
Observa-se que os serviços estudados funcionam fechados em si mesmos, não existindo um sistema de referência para outros serviços nem tampouco contra-referências registradas em prontuário, fato este que dificulta a integralidade da assistência.

QUADRO 2. Diagnósticos mais Freqüentes por Unidade, Estudo de Demanda, Outubro de 1990 a Setembro de 1991

\begin{tabular}{|lc|lc|lc|}
\hline \multicolumn{2}{|c|}{ CMS } & & \multicolumn{2}{c|}{ PAM } & \multicolumn{2}{c|}{ PU } \\
\hline Diagnóstico & $\%$ & Diagnóstico & $\%$ & Diagnóstico & $\%$ \\
\hline Ira-Leve & 11,4 & Hipertensão & 9,8 & Externas & 11,3 \\
Hipertensão & 8,8 & Ira-Leve & 7,5 & Ira-Leve & 10,7 \\
Piodermites & 5,2 & Pré-Natal & 6,5 & Asma & 8,5 \\
Ira-Moderada & 4,8 & Ansiedade & 6,1 & Hipertensão & 6,2 \\
Colpite/Vaginite & 4,0 & Colpite/Vaginite & 5,7 & Dengue & 6,0 \\
Parasitoses & 3,8 & Asma & 5,2 & Ira-Moderada & 5,4 \\
Tuberculose & 3,4 & Parasitoses & 4,5 & Diarréia & 5,2 \\
Escabiose/Pediculose & 3,4 & Puericultura & 3,5 & Virose & 3,9 \\
Eczemas & 2,9 & Preventivo & 3,3 & Cefaléia & 2,9 \\
Diarréia & 2,5 & Dor Abdominal & 2,5 & Febre & 2,1 \\
\hline Outras & 49,8 & Outras & 45,4 & Outras & 37,7 \\
\hline
\end{tabular}

\section{RESUMO}

CARVALHO, M. S.; d'ORSI, E.; PRATES, E. C.; TOSCHI, W. D. M.; SHIRAIWA, T.; CAMPOS, T. P.; ELL, E.; GARCIA, N. L.; JUNQUEIRA, A. P.; SERRÃO, S. A. \& TAVARES, E. L. Demanda Ambulatorial em Três Serviços da Rede Pública do Município do Rio de Janeiro, Brasil. Cad. Saúde Públ., Rio de Janeiro, 10(1): 17-29, jan/mar, 1994.

A fim de se conhecer o perfil demográfico e nosológico da clientela, bem como avaliar a qualidade dos registros das consultas em prontuário, realizou-se um estudo de demanda em três serviços da rede pública - dois ambulatoriais e um de urgência - localizados na X Região Administrativa do município do Rio de Janeiro, Estado do Rio de Janeiro, Brasil. A amostra obtida foi de 2.029 pacientes, com 3.980 consultas realizadas no período de 1 de outubro de 1990 a 30 de setembro de 1991. Os diagnósticos foram codificados pela $9^{\mathrm{a}}$ revisão da Classificação Internacional de Doenças. Cerca de $60 \%$ dos atendimentos foram prestados a mulheres, mais de metade entre 15 e 49 anos. Crianças menores de 10 anos representaram $28 \%$ do total de atendimentos. Grande parte da demanda (38\%) é formada pela população favelada da área. A qualidade dos registros da consulta em prontuário foi precária, faltando importantes informações. Os diagnósticos mais freqüentes foram classificados no capítulo XVI - afecções maldefinidas -, seguindo-se as doenças respiratórias, infecciosas, geniturinárias e circulatórias. Nos serviços ambulatoriais foi grande a procura por ações preventivas, como consulta de prénatal e puericultura, inclusive na unidade que historicamente realizava apenas ações curativas. No serviço de urgência predominou $o$ atendimento por causas externas. Entre os diagnósticos específicos mais freqüentes estão 
as infecções respiratórias agudas, a hipertensão arterial, problemas ginecológicos, consultas de pré-natal e doenças de pele. Não há registro de referência para outros serviços. A precariedade do registro médico traz prejuízos à assistência. A utilização destes registros, ainda que criticamente, é fundamental para melhorá-los.

Palavras-Chave: Demanda Ambulatorial; Morbidade; Qualidade de Registros

\section{REFERÊNCIAS BIBLIOGRÁFICAS}

ASHTON-TATE, 1985. Dbase III plus. São Paulo: Datalógica.

BENITEZ, G. \& MONZÓ, L., 1985. Morbilidad en consultas ambulatoriales del Policlínico de la Comunidad "Belkys Sotomayor", Año 1983. Revista Cubana de Administración en Salud, 1985: 234-242.

CARVALHO, M. S.; GIFFIN, K. \& SHIRAIWA,T. (Org.), 1993a. Condições de Vida e Morte em Populações Urbanas - Uma Proposta para os Serviços de Saúde. Rio de Janeiro: Ensp/SDE. (Panorama Ensp, 5)

CARVALHO, M. S.; d'ÓRSI, E.; MELO, E. C. P \& CAMPOS, T. P., 1993b. Estudo de Demanda Ambulatorial: do planejamento à divulgação dos resultados. Rio de Janeiro: Ensp/SDE/Pares.

DEAN, A. G.; DEAN, J. A.; BURTON, A. H. \& DICKER, R. C., 1990. Epi Info, Version 5: a Word Processing, Database and Statistics Program for Epidemiology on Micro-Computers. Atlanta: Centers for Disease Control.

DELOZIER, J. E. \& GAGNON, R. O., 1991. National Ambulatory Medical Care Survey: 1989 Summary, Advance Data from Vital and Health Statistics of the National Center for Health Statistics. Washington DC: National Center for Health Statistics. (Mimeo.)

FIBGE (Fundação Instituto Brasileiro de Geografia e Estatística), 1980. Censo Demográfico de 1980. Dados distritais: Rio de Faneiro, 1983. Volume 1, T. 17, Rio de Janeiro: IBGE. $\left(8^{\circ}\right.$ Recenseamento Geral do Brasil)
, 1991. Censo Demográfico de 1991. Resultados preliminares divulgados em 1992. Rio de Janeiro: IBGE. (Mimeo.)

IPLANRIO (Instituto de Planejamento Municipal do Rio de Janeiro), 1982. Cadastro de favelas do Município do Rio de Janeiro. Rio de Janeiro: Iplanrio. (Mimeo.)

GUIA QUATRO RODAS, 1990. Guia Rio. São Paulo: Abril.

KOSTRZEWSKI, J., 1982. Visitas médicas y morbilidad en Polonia, julio 1967-junio 1968. In: Mediociones de los Niveles de Salud (H. Ipsen \& J. Kostrzewski, eds.), pp. 283-294, Barcelona: Salvat Editores.

MS (Ministério da Saúde), 1984. Assistência Integral à Saúde da Criança: Ações básicas. Brasília: Centro de Documentação do Ministério da Saúde.

, 1988. Normas Técnicas para o Programa Nacional de Educação e Controle da Hipertensão Arterial (PNECHA). Brasília: Secretaria Nacional de Programas Especiais de Saúde, Centro de Documentação do Ministério da Saúde.

OMS (Organização Mundial da Saúde), 1978. Manual de Classificação Internacional de Doenças, Lesões e Causas de Óbito. 9a Revisão - 1975. Genebra: OMS.

RADAELLI, S. M.; TAKEDA, S. M. P.; GIMENO, L. I. D.; WAGNER, M. B.; KANTER, F. J.; MELLO, V. M.; BORGES, J. C. \& DUNCAN, B. B., 1990. Demanda de serviço de saúde comunitária na periferia de área metropolitana. $R e$ vista de Saúde Pública, 24: 232-240.

SPC (Software Publishing Corporation), 1990. Harvard Graphics, Version 2.3 Mountains View: SPC.

TANAKA, O. Y. \& ROSENBURG, C. P., 1990. Análise da utilização pela clientela de uma unidade ambulatorial da Secretaria da Saúde do Município de São Paulo, SP (Brasil). Revista de Saúde Pública, 24: 60-68.

YAZZLE-ROCHA, J. S. \& NOGUEIRA, J. L., 1985. Padrões de morbidade em assistência primária na região de Ribeirão Preto, SP (Brasil). Revista de Saúde Pública, 19: 215-224. 